\title{
Transverse mode coupling instability threshold with space charge and different wakefields
}

\author{
V. Balbekov* \\ Fermi National Accelerator Laboratory, P.O. Box 500, Batavia, Illinois 60510, USA
}

(Received 23 August 2016; published 10 March 2017)

\begin{abstract}
Transverse mode coupling instability of a single bunch with space charge (SC) and a wakefield is considered within the framework of the boxcar model. Eigenfunctions of the bunch without a wake are used as a basis for the solution of the equations with the wakefield included. A dispersion equation for a constant wake is presented in the form of an infinite continued fraction and also as the recursive relation with an arbitrary number of basis functions. Realistic wakefields are considered as well including resistive wall, square, and oscillating wakes. It is shown that the transverse mode coupling instability threshold of the negative wake grows in absolute value when the SC tune shift increases. The threshold of the positive wake goes down at increasing the SC tune shift. The explanation is developed by an analysis of the bunch spectrum.
\end{abstract}

DOI: 10.1103/PhysRevAccelBeams.20.034401

\section{INTRODUCTION}

Transverse mode coupling instability (TMCI) has been observed first in PETRA and explained by Kohaupt on the base of the two-particle model [1]. A lot of papers on this subject have been published later, including handbooks and surveys (see, e.g., [2]). It is established that the instability occurs as a result of a coalescence of the neighboring headtail tunes caused by the bunch wakefield.

TMCI with space charge (SC) has been considered first by Blaskiewicz [3]. The main point of this paper is that the SC pushes up the TMCI threshold, that is, improves the beam stability. However, a nonmonotonic dependence of the TMCI threshold and rate on the SC tune shift has been sometimes demonstrated in Ref. [3]. It followed from several examples that the stability and instability areas can change each other when the SC tune shift increases. The results have been confirmed later by the same author with the help of a numerical simulation of the instability at a modest magnitude of the SC tune shift [4].

The so-called three-mode model has been developed in Ref. [5] for an analytical description of the TMCI with space charge, chromaticity, and an arbitrary wake. This simple model confirms that the TMCI threshold of negative wakes goes up in modulus when the SC tune shift increases. However, only the case of modest SC has been investigated in Ref. [5], though the proposed equations allow one to suggest that a sudden kink of the threshold curve is possible at the higher shift. Therefore,

\footnotetext{
*balbekov@fnal.gov

Published by the American Physical Society under the terms of the Creative Commons Attribution 3.0 License. Further distribution of this work must maintain attribution to the author(s) and the published article's title, journal citation, and DOI.
}

the field of application of the three-mode model is still an open question.

The case of very high space charge has been considered in Refs. [6,7]. It was confirmed in both papers that the space charge heightens the TMCI threshold until the ratio of the SC tune shift to the synchrotron tune reaches the border in several tens or a hundred units. However, the authors have expressed different opinions about the further behavior of the threshold. As it follows from Ref. [7], the threshold growth should continue at higher SC as well. On the contrary, it was suggested in Ref. [6] that the threshold growth can cease and turn back over the mentioned border.

The last statement has been supported recently in Ref. [8]. I have used the known eigenfunctions of the boxcar bunch [9] to get a convenient basis for investigation of the TMCI problem in depth. However, a disclosure of some errors at the numerical solutions of the obtained equations forces me to revise the conclusions. The equations are recomputed in the present paper at any value of the SC tune shift and different wakes including the resistive wall, square, and oscillating ones. The increase of the TMCI threshold by the SC is observed in all the cases.

\section{BASIC EQUATIONS AND ASSUMPTIONS}

The terms, basic symbols, and equations of Ref. [5] are used in this paper. In particular, linear synchrotron oscillations are considered here being characterized by amplitude $A$ and phase $\phi$ or by the corresponding Cartesian coordinates:

$$
\theta=A \cos \phi, \quad u=A \sin \phi .
$$

Thus, $\theta$ is the azimuthal deviation of a particle from the bunch center in the rest frame, and variable $u$ is proportional to the momentum deviation about the bunch central 
momentum (the proportionality coefficient plays no part in this paper). A coherent transverse displacement of the particles in some point of the longitudinal phase space will be presented as the real part of the function

$$
X(A, \phi, t)=Y(A, \phi) \exp \left[-i\left(Q_{0}+\zeta\right) \theta-i\left(Q_{0}+\nu\right) \Omega_{0} t\right],
$$

where $\Omega_{0}$ is the revolution frequency, $Q_{0}$ is the central betatron tune, and $\nu$ is the tune addition produced by the space charge and wakefield. Generally, $\zeta$ is the normalized chromaticity; however, only the case $\zeta=0$ will be investigated in this paper. Then the function $Y$ satisfies the equation $[5,7]$

$$
\begin{aligned}
\nu Y & +i Q_{s} \frac{\partial Y}{\partial \phi}+\Delta Q(\theta)[Y(\theta, u)-\bar{Y}(\theta)] \\
& =2 \int_{\theta}^{\infty} q\left(\theta^{\prime}-\theta\right) \bar{Y}\left(\theta^{\prime}\right) \rho\left(\theta^{\prime}\right) d \theta^{\prime},
\end{aligned}
$$

where $F(\theta, u)$ and $\rho(\theta)$ are the normalized distribution function and corresponding linear density of the bunch, respectively, $Q_{s}$ is the synchrotron tune, $\Delta Q(\theta) \propto \rho(\theta)$ is the space charge tune shift, and $\bar{Y}(\theta)$ is the bunch displacement in the real space which can be found by means of the formula

$$
\rho(\theta) \bar{Y}(\theta)=\int_{-\infty}^{\infty} F(\theta, u) Y(\theta, u) d u
$$

The function $q(\theta)$ is proportional to the usual transverse wakefield function $W_{1}(z)$ :

$$
q(\theta)=\frac{r_{0} R N_{b} W_{1}(-R \theta)}{8 \pi \beta \gamma Q_{0}}
$$

with $r_{0}=e^{2} / m c^{2}$ as the classic radius of the particle, $R$ as the accelerator radius, $N_{b}$ as the bunch population, and $\beta$ and $\gamma$ as the normalized velocity and energy, respectively [2].

A solution of Eq. (3) can be found by its expansion in terms of the eigenfunctions of the corresponding homogeneous equation, which is

$$
\nu_{j} Y_{j}+i Q_{s} \frac{\partial Y_{j}}{\partial \phi}+\Delta Q(\theta)\left[Y_{j}(\theta, u)-\bar{Y}_{j}(\theta)\right]=0 .
$$

It is easy to check that the functions form an orthogonal basis with the weight function $F(\theta, u)$. Besides, we will impose the normalization condition:

$$
\iint F(\theta, u) Y_{j}^{*}(\theta, u) Y_{k}(\theta, u) d \theta d u=\delta_{j k},
$$

where the star denotes complex conjugation. Then, looking for the solution of Eq. (3) in the form

$$
Y=\sum_{j} C_{j} Y_{j}
$$

one can get the expression for the unknown coefficients $C_{j}$ :

$\sum_{j}\left(\nu-\nu_{j}\right) C_{j} Y_{j}=2 \sum_{j} C_{j} \int_{\theta}^{\infty} \bar{Y}_{j}\left(\theta^{\prime}\right) \rho\left(\theta^{\prime}\right) q\left(\theta^{\prime}-\theta\right) d \theta^{\prime}$,

where $\bar{Y}_{j}$ and $Y_{j}$ are also connected by Eq. (4). Multiplying Eq. (9) by the factor $F(\theta, u) Y_{J}^{*}(\theta, u)$, integrating over $\theta$ and $u$, and using normalization condition (7), one can get the series of equations for the coefficients $C_{j}$ :

$$
\begin{aligned}
\left(\nu-\nu_{J}\right) C_{J}= & 2 \sum_{j} C_{j} \int_{-\infty}^{\infty} \rho(\theta) \bar{Y}_{J}^{*}(\theta) d \theta \\
& \times \int_{\theta}^{\infty} \rho\left(\theta^{\prime}\right) \bar{Y}_{j}\left(\theta^{\prime}\right) q\left(\theta^{\prime}-\theta\right) d \theta^{\prime}
\end{aligned}
$$

\section{BOXCAR MODEL}

The boxcar model is characterized by the following expressions for the bunch distribution function and its linear density:

$$
\begin{aligned}
F & =\frac{1}{2 \pi \sqrt{1-A^{2}}}=\frac{1}{2 \pi \sqrt{1-\theta^{2}-u^{2}}}, \\
\rho(\theta) & =\frac{1}{2} \quad \text { at }|\theta|<1 .
\end{aligned}
$$

Because the eigenfunctions depend on two variables $(\theta-u)$ (or $A-\phi)$, it is more convenient to represent $j$ as a pair of the indexes:

$$
j \equiv\{n, m\}, \quad Y_{j} \equiv Y_{n, m} .
$$

Analytical solutions of Eq. (6) for the boxcar bunch have been found by Sacherer [9]. The most important point is that the averaged eigenfunctions $\bar{Y}_{n, m}$ do not depend on the second index being proportional to the Legendre polynomials: $\bar{Y}_{n, m}(\theta)=\bar{Y}_{n}(\theta) \propto P_{n}(\theta), n=0,1,2, \ldots$ At any $n$, there are $n+1$ different eigenmodes $Y_{n, m}(\theta, u)$ satisfying the equation

$$
\left(\nu_{n, m}+\Delta Q\right) Y_{n, m}+i Q_{s} \frac{\partial Y_{n, m}}{\partial \phi}=\Delta Q S_{n, m} P_{n}(\theta),
$$

where $m=n, n-2, \ldots,-n$. The space charge tune shift $\Delta Q$ is constant in this model, and the coefficients $S_{n, m}$ are added to the right-hand part of the equation to meet the 


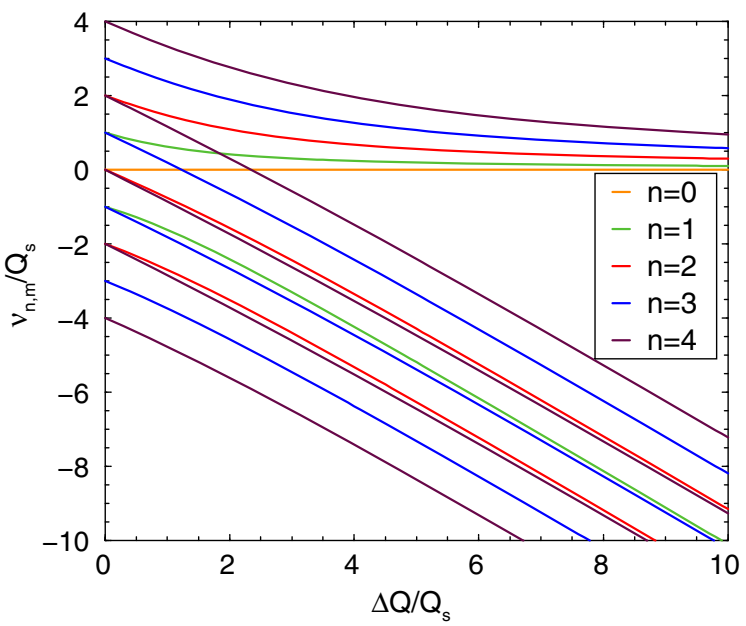

FIG. 1. Eigentunes of the boxcar bunch without a wake. At any $n$, there are $n+1$ eigentunes starting at $\Delta Q=0$ from the points $\nu_{n, m}=m Q_{s}, m=n, n-2, \ldots,-n$.

normalization condition given by Eq. (7). Details of these calculations are placed in the Appendix, and several important examples are represented in Figs. 1 and 2.

The dependence of the eigentunes on the SC tune shift is shown in Fig. 1. It is seen that all of them start at $\Delta Q=0$ from the points $\nu_{n, m}(0)=m Q_{s}$. It is the commonly accepted convention to use the term "multipole" for the collective synchrotron oscillations of such a frequency; that is, the index $m$ should be treated here as the multipole number. Another index $n$ characterizes the eigenfunction power. This feature is normally associated with a radial mode number, the lower power corresponding to the lower number. Because $n \geq|m|$ in this case, the mode $\{|m|, m\}$ should be treated as the lowest radial mode of the $m$ th multipole.

At $\Delta Q \neq 0$, the multipoles mix together, and the eigentunes split into two groups. In the first of them, all

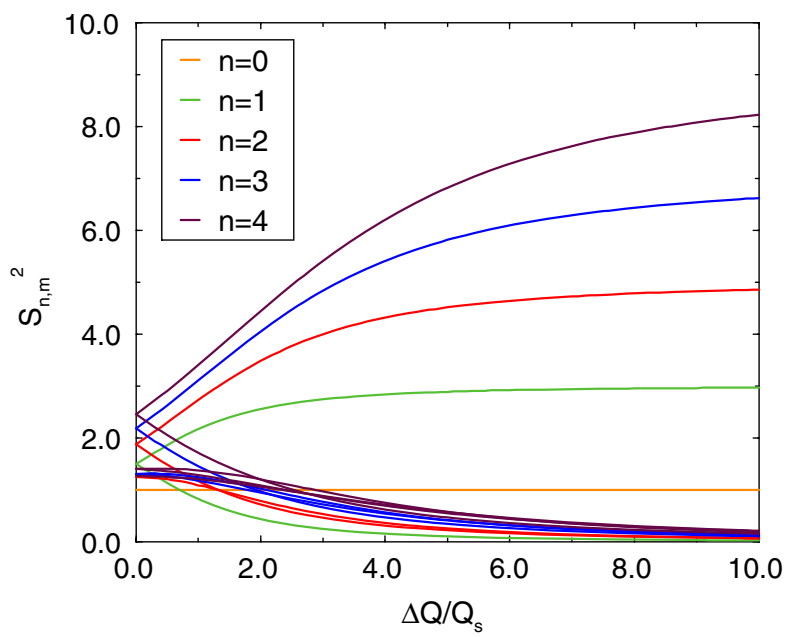

FIG. 2. Normalizing coefficients of the boxcar bunch. The rising lines refer to the case $m=n$. the tunes have a positive value which tends to 0 at $\Delta Q / Q_{s} \rightarrow \infty$. By the origin, all of them are the lowest radial modes $\{n, n\}$. The corresponding normalizing coefficients $S_{m, m}^{2} \rightarrow 2 n+1$ at $\Delta Q / Q_{s} \rightarrow \infty$ (Fig. 2). In the second group, the tunes are about $\nu_{n, m} \simeq m Q_{s}-\Delta Q$, being weakly dependent on the radial index $n$. The normalizing coefficients tend to 0 in this group.

Note that the transient conjugations of some eigentunes (the line crossing in Fig. 1) is not evidence of the TMCI instability in the case, because the corresponding eigenfunctions are orthogonal and uncoupled.

With $\bar{Y}_{n, m}=S_{n, m} P_{n}(\theta)$, the series (10) for the boxcar bunch obtains the form

$$
\left(\nu-\nu_{N, M}\right) C_{N, M}=q_{0} S_{N, M}^{*} \sum_{n=0}^{\infty} R_{N, n} \sum_{m} S_{n, m} C_{n, m}
$$

with the matrix

$$
R_{N, n}=\frac{1}{2} \int_{-1}^{1} P_{N}(\theta) d \theta \int_{\theta}^{1} P_{n}\left(\theta^{\prime}\right) w\left(\theta^{\prime}-\theta\right) d \theta^{\prime}
$$

The notation

$$
q(\theta)=q_{0} w(\theta)
$$

is used here and later to separate the wake strength from its form and to furnish the relation

$$
R_{0,0}=\frac{1}{2} \int_{0}^{2}(2-\theta) w(\theta) d \theta=1 .
$$

Besides, we will use the designations

$$
\begin{aligned}
Z_{n} & =\sum_{m} S_{n, m} C_{n, m}, \\
W_{n}(\nu) & =\sum_{m} \frac{\left|S_{n, m}\right|^{2}}{\nu-\nu_{n, m}} .
\end{aligned}
$$

Then series (14) obtains the most compact form:

$$
Z_{N}=q_{0} W_{N} \sum_{n=0}^{\infty} R_{N, n} Z_{n}
$$

\section{CONSTANT WAKE}

Several realistic examples of the wake will be considered in Sec. V. However, the simplest model is preliminarily investigated in this section to discover the main features of the effect. This simulative wake has a constant value within the bunch and decays after it; that is, $w(\theta)=1$ in 
TABLE I. Fragment of the matrix $R_{N, n}$. Its general form is given by Eqs. (13) and (16).

\begin{tabular}{lcccccc}
\hline \hline$n \rightarrow$ & 0 & 1 & 2 & 3 & 4 & 5 \\
\hline$N=0$ & 1 & $1 / 3$ & 0 & 0 & 0 & 0 \\
$N=1$ & $-1 / 3$ & 0 & $1 / 15$ & 0 & 0 & 0 \\
$N=2$ & 0 & $-1 / 15$ & 0 & $1 / 35$ & 0 & 0 \\
$N=3$ & 0 & 0 & $-1 / 35$ & 0 & $1 / 63$ & 0 \\
$N=4$ & 0 & 0 & 0 & $-1 / 63$ & 0 & $1 / 99$ \\
$N=5$ & 0 & 0 & 0 & 0 & $-1 / 99$ & 0 \\
\hline \hline
\end{tabular}

Eqs. (15)-(17). Though the wakes are negative in most cases [2], a positive wake is possible as well, and it was observed in practice $[10,11]$. Therefore, both signs of the parameter $q_{0}$ are analyzed in this section.

It is easy to verify that, at $w=1$ and $N \neq 0$, the matrix $R_{N, n}$ is

$R_{N, n}=-\frac{\delta_{N-1, n}}{(2 N-1)(2 N+1)}+\frac{\delta_{N+1, n}}{(2 N+1)(2 N+3)}$

(its small fragment is shown in Table I).

A series of linear equations like Eq. (19) is resolvable if its determinant is zero: $D(\nu)=0$. It is easy to check the relations $\nu_{0,0}=0, S_{0,0}=1$, that is, $W_{0}=1 / \nu$. Using these features and applying the mathematical induction method, one can represent the resolvability condition of series (19), that is, the dispersion equation for the bunch eigentunes, in terms of an infinite continued fraction

$$
D(\nu)=\nu-q_{0}+\frac{\left(q_{0} / 3\right)^{2} W_{1}}{1+\frac{\left(q_{0} / 15\right)^{2} W_{1} W_{2}}{1+\frac{\left(q_{0} / 35\right)^{2} W_{2} W_{3}}{1+\ldots}}}=0 .
$$

This expression has to be truncated in reality by applying the assumption $W_{n}=0$ at $n \geq n_{\max }$. Assigning the truncated left-hand part of Eq. (21) as $D_{n_{\max }}(\nu)$ and applying again the mathematical induction method, one can write the approximate dispersion equation as

$$
D_{n_{\max }}(\nu)=0
$$

with the following recurrent relations:

$$
D_{n}=D_{n-1}+D_{n-2} \frac{q_{0}^{2} W_{n-1} W_{n}}{\left(4 n^{2}-1\right)^{2}} \quad(n \geq 2) .
$$

It is shown in Eq. (A15) of the Appendix that

$$
W_{1}(\nu)=\frac{3(\nu+\Delta Q)}{\nu(\nu+\Delta Q)-Q_{s}^{2}} .
$$

Therefore, the initial conditions of the series (23) are

$$
\begin{aligned}
& D_{0}=\nu-q_{0}, \\
& D_{1}=\nu-q_{0}+\left(\frac{q_{0}}{3}\right)^{2} \frac{3(\nu+\Delta Q)}{\nu(\nu+\Delta Q)-Q_{s}^{2}} .
\end{aligned}
$$

\section{A. Three-mode approximation}

Equation (22) is trivial at $n_{\max }=0: D_{0}(\nu)=0$ means $\nu=q_{0}$, as it follows from Eq. (25a). It describes the wake contribution to the tune of the lowest (rigid) head-tail mode of the bunch. Of course, the TMCI cannot appear in this approximation, and the simplest equation to disclose it is $D_{1}(\nu)=0$; that is, in accordance with Eq. (25b),

$$
(\nu-q)\left(\nu-\frac{Q_{s}^{2}}{\nu+\Delta Q}\right)=-\frac{q_{0}^{2}}{3}
$$

This third-order equation exactly coincides with Eq. (7.3) of Ref. [5] (without chromaticity) despite the fact that very different concepts have been used to derive them. However, the examples presented in Ref. [5] have been restricted by the modest SC tune shift: $\Delta Q / Q_{s}<3$. The situation beyond this region will be explored here for the best understanding of the phenomenon and for further development of the technique.

The imaginary part of a solution of Eq. (26) is plotted in Fig. 3 against the wake strength at different SCs. According to the plot, the instability threshold is $\left|q_{\text {th }} / Q_{s}\right|=0.567$ at $\Delta Q=0$ (black lines). Its dependence on $\Delta Q / Q_{s}$ is shown in Fig. 4. The plot is very simple at $q_{0}>0$ : the threshold goes down monotonically, tending to 0 when the space charge increases. The case of a negative wake is more complicated and requires a special comment. The absolute

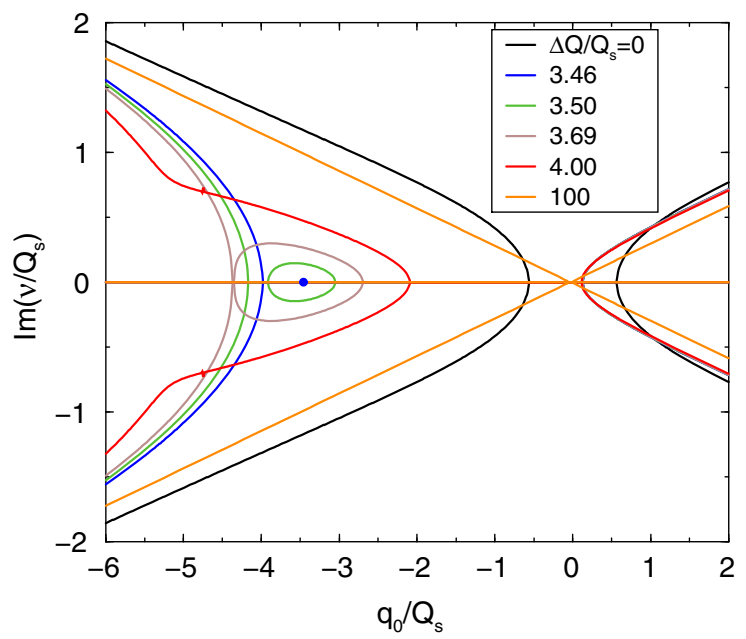

FIG. 3. Imaginary part of the boxcar eigentunes against the wake strength at different values of the space charge tune shift. There are two regions of instability if the wake is negative and $3.46<\Delta Q / Q_{s}<3.69$. 


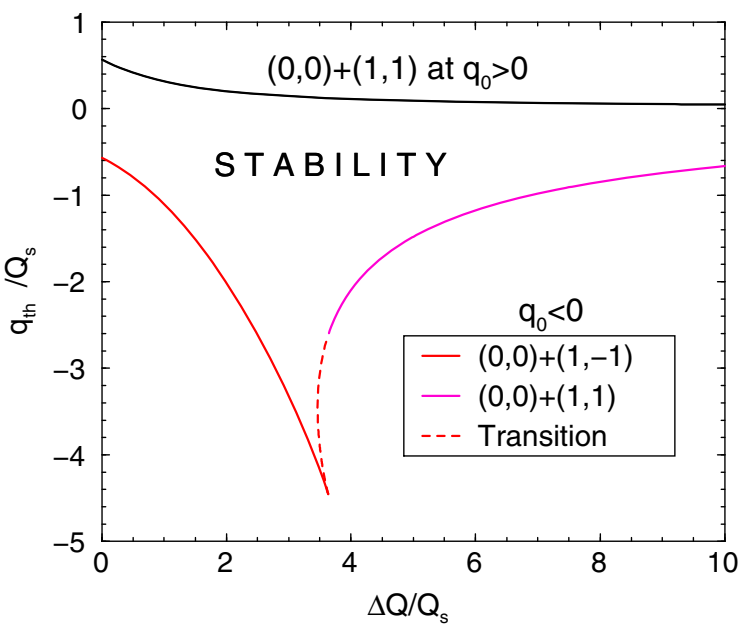

FIG. 4. Instability threshold of the boxcar bunch against the SC tune shift (positive and negative wakes). Indexes of the coalesced modes are shown for each part of the threshold line.

value of its threshold increases with $\Delta Q$, reaching $q_{\mathrm{th}} / Q_{s} \simeq-4$ at $\Delta Q / Q_{s}=3.46$ (blue parabola in Fig. 3). The picture crucially changes after that, because a new region of instability arises whose initial position is shown as the blue point in Fig. 3. Then it quickly expands, stepping through the green oval to the brown one and joining with the primary region of instability (brown parabola) at $\Delta Q / Q_{s}=3.69$. The barrier between the parts tears at higher $\Delta Q$, resulting in a single region of instability (red). Its right-hand border goes to the right- that is, the TMCI threshold goes down-if the SC tune shift continues to grow.

\section{B. Higher approximations}

Higher approximations should be involved to validate the three-mode model, to establish its applicability limit, and to go beyond it.

The first step in this way is an investigation of the equation $D_{2}(\nu)=0$. According to Eq. (23), its expanded form is

$\nu-q_{0}+\frac{q_{0}^{2} W_{1}(\nu)}{9}+\frac{q_{0}^{2}\left(\nu-q_{0}\right) W_{1}(\nu) W_{2}(\nu)}{225}=0$,

where $W_{1}(\nu)$ is given by Eq. (24) and

$$
W_{2}(\nu)=\frac{\left|S_{2,-2}\right|^{2}}{\nu-\nu_{2,-2}}+\frac{\left|S_{2,0}\right|^{2}}{\nu-\nu_{2,0}}+\frac{\left|S_{2,2}\right|^{2}}{\nu-\nu_{2,2}} .
$$

The required parameters have to be obtained by the solution of Eq. (13) with $n=2$ as described in the Appendix. With the notations $\nu_{n, m}=\hat{\nu}_{n, m} Q_{s}-\Delta Q$, the eigentunes appear as all roots of the dispersion equation

$$
\begin{aligned}
\hat{\nu}_{2, m}\left(\hat{\nu}_{2, m}^{2}-4\right) & =\frac{\Delta Q}{Q_{s}}\left(\hat{\nu}_{2, m}^{2}-1\right), \\
\left|S_{2, m}\right|^{2} & =\frac{5\left(\hat{\nu}_{2, m}^{2}-1\right)^{2}}{\hat{\nu}_{2, m}^{4}+\hat{\nu}_{2, m}^{2}+4} .
\end{aligned}
$$

The substitution of the functions $W_{1-2}(\nu)$ into Eq. (27) results in the equation of sixth power:

$$
\begin{aligned}
(\nu & \left.-q_{0}\right)\left(\nu-\frac{Q_{s}^{2}}{\nu+\Delta Q}\right)+\frac{q_{0}^{2}}{3} \\
& =-\frac{q_{0}^{2}(\nu-q)}{75}\left(\frac{\left|S_{2,-2}\right|^{2}}{\nu-\nu_{2,-2}}+\frac{\left|S_{2,0}\right|^{2}}{\nu-\nu_{2,0}}+\frac{\left|S_{2,+2}\right|^{2}}{\nu-\nu_{2,+2}}\right) .
\end{aligned}
$$

This equation has six roots, which are different real numbers inside the stability area. However, at least two of them should be coinciding at the border of this area, which feature can be used for the search of the instability threshold. The corresponding threshold of the negative wake is presented in Fig. 5 by the magenta line. The case $q_{0}>0$ is not considered in this subsection, because the result almost does not depend on $n_{\max }$ and can be reasonably described by the three-mode approximation, Eq. (25).

A similar method can be used for the analysis of higher approximations, though the corresponding formulas are essentially more cumbersome. Generally, the case involves $\left(n_{\max }+1\right)\left(n_{\max }+2\right) / 2$ basis vectors and leads to an algebraic equation of the same power, where $n_{\max }$ is the order of the highest used Legendre polynomial.

The results of the calculations are collected in Fig. 5 at $n_{\max }=1-12$ (dispersion equation of $3-91$ power). It is

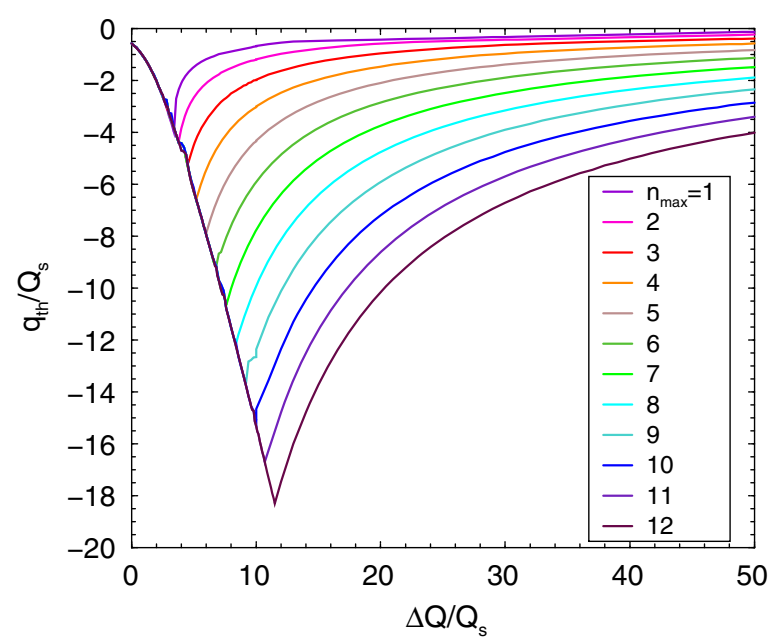

FIG. 5. Threshold curve of the boxcar bunch in different approximations. The index $n_{\max }$ means maximal power of the Legendre polynomial in the expansion. The left-hand line shows the TMCI threshold, and the right-hand rising lines are unphysical because of an absence of the convergence. 
seen that, at a rather small $\mathrm{SC}$, the absolute value of the threshold rises with $\Delta Q$, different approximations provide actually coinciding results in their region of applicability, and each additional step simply expands this region. For example, the three-mode approximation $\left(n_{\max }=1\right)$ provides the correct magnitude of the threshold at $\Delta Q / Q_{s} \leq 3.46$, but at least $n_{\max }=12$ (91-mode approximation) is needed to get proper results within the range $\Delta Q / Q_{s}=0-12$.

The sequential decrease of the threshold cannot be treated as a physical effect because of the absence of the convergence. The opposite assumption which has been admitted in Ref. [8] was coming from an insufficient accuracy of numerical calculations which has led to an incomplete separation of numerous and very tightly spaced radial modes.

\section{The bunch spectrum}

The inadequate convergence of the curves in Fig. 5 remains the open question: which is the TMCI threshold of the negative wake at a very large magnitude of $\Delta Q / Q_{s}$, such as several tens or more? There is related information in Ref. [7]: at such conditions, the TMCI cannot be caused by a coalescence of positive eigentunes of the bunch. The last reservation is important, because only a part of the boxcar modes has been used for the analysis in Ref. [7]. Tunes of these modes are located in the upper part of Fig. 1. Therefore, a more detailed examination of the bunch spectrum is needed with the wake to check the results, including all bunch tunes $\nu_{\text {th }}(\Delta Q)$ at the frontier of the TMCI area.

The very first example of this has been given in Fig. 4, where indexes of the coalesced modes are specified for the three-mode approximation. The more detailed view is represented in Fig. 6, where the full spectrum of the bunch is plotted at $n_{\max }=5$ (21-mode approximation). The most important spectral lines are displayed by special colors: blue for the modes $\{0,0\}$ and $\{1,-1\}$ and red for the modes $\{5,5\}$ and $\{4,4\}$. According to the picture, the coalescence of these modes is responsible for the TMCI at $\Delta Q / Q_{s}<6$ or $>6$, correspondingly. Just switching from the lower pair to the upper one causes the sharp kink of the threshold curve, which is shown in the plot by a dashed line. An incidental interference of other modes slightly affects the curve but does not change its general contour.

Other examples are given in Fig. 7, where the most important spectral lines are plotted at three different approximations: $n_{\max }=6,9,12$. The lower curves represent tunes of the modes $\{0,0\}+\{1,-1\}$, which are coalesced in the beginning and are closely located later. The convergence manifests itself in the fact that the coalesced part of the curves expands when $n_{\max }$ increases.

The upper curves in Fig. 7 represent the tunes

$$
\left\{n_{\max }, n_{\max }\right\}+\left\{n_{\max }-1, n_{\max }-1\right\} .
$$

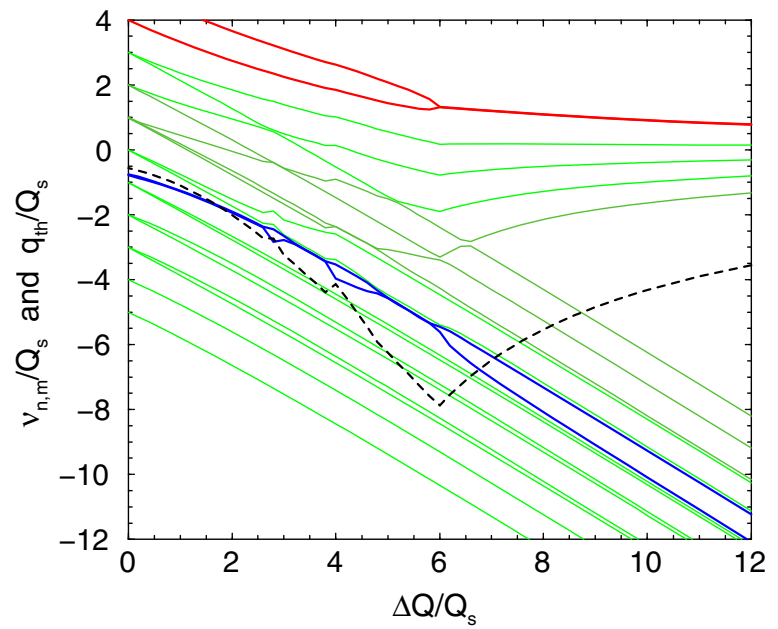

FIG. 6. The bunch spectrum in the TMCI frontier at $n_{\max }=5$ (21-mode approximation). The most important modes are shown by blue and red lines. The TMCI threshold is represented by the dashed black line.

It is seen that the lines of the same color merge at rather large $\Delta Q / Q_{s}$. However, it is a divergent process, because the junction point does not tend to a certain limit when $n_{\max }$ increases. It inevitably leads to the conclusion that the junction of the positive tunes in Figs. 6 and 7, as well as the associated leap of the threshold, is not a physical effect. The engaging of Ref. [7] allows one to assert that this statement should be true in any approximation.

Therefore, a monotonic rising of the TMCI threshold looks like the most credible assumption. It also complies with the behavior of the low crucial modes, which are

$\nu_{0,0} \sim q_{0}, \quad \nu_{1,-1} \simeq-Q_{s}-\Delta Q$.

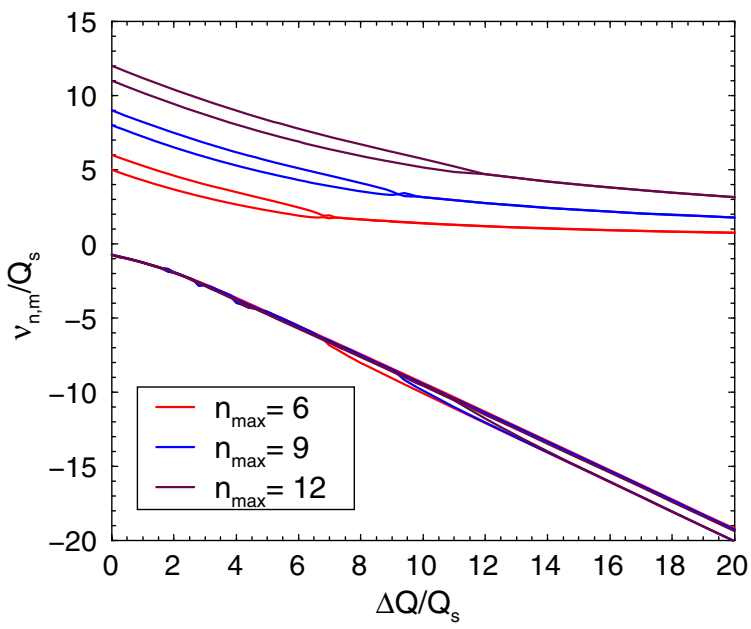

FIG. 7. The most important spectral lines in different approximations. Lower lines, modes $\{0,0\}$ and $\{1,-1\}$; upper ones, the highest observed tunes allowed by considering approximation. 
TABLE II. Fragment of the matrix $R_{N, n}$ for a resistive wake.

\begin{tabular}{|c|c|c|c|c|c|c|}
\hline$n \rightarrow$ & 0 & 1 & 2 & 3 & 4 & 5 \\
\hline$N=0$ & 1 & 0.20000 & -0.02857 & 0.00952 & -0.00433 & 0.00233 \\
\hline$N=1$ & -0.20000 & 0.14286 & 0.06667 & -0.01299 & 0.00513 & -0.00260 \\
\hline$N=2$ & -0.02857 & -0.06667 & 0.06494 & 0.03590 & 0.00779 & 0.00332 \\
\hline$N=3$ & -0.00952 & -0.01299 & -0.03590 & 0.03896 & 0.02323 & -0.00533 \\
\hline$N=4$ & -0.00433 & -0.00513 & -0.00779 & -0.02323 & 0.02666 & 0.01659 \\
\hline$N=5$ & -0.00233 & -0.00260 & -0.00332 & -0.00533 & -0.01659 & 0.01970 \\
\hline
\end{tabular}

According to this, the instability condition $\nu_{0,0}=\nu_{1,-1}$ should result in the threshold relation $q_{\mathrm{th}} \sim-\Delta Q$.

\section{TMCI WITH A REALISTIC WAKE}

The series of equations (19) with matrix $R_{N, n}$ given by Eq. (15) is applicable at any wake function $q(\theta)=q_{0} w(\theta)$. However, dispersion equation (21) and its approximate forms provided by Eqs. (22)-(24) are valid only with a constant wake. Therefore, more standard procedures should be generally used for the solution of Eq. (19). It results in some deterioration of the accuracy and compels one to restrict the number of used basis vectors. Performed calculations with a constant wake attest that the "old" and the "new" results coincide at $n_{\max } \leq 9$ (55-mode approximation); otherwise, some real roots can be lost. This restriction is accepted below at the calculations with realistic wakes.

\section{A. Resistive wall wake}

Resistive wall impedance is the most general and important source of transverse instabilities in circular accelerators. The corresponding wake function has a maximum at $z \simeq-b / \gamma$, where $b$ is the beam pipe radius and $z$ is the distance from the field source to the observation point. If the bunch length $z_{b}$ satisfies the condition $z_{b} \gg b / \gamma$ and the pipe wall is rather thick, the simplest expression for the resistive wake function can be used [2]:

$$
W_{1}(z)=-\frac{4 R}{b^{3}} \sqrt{\frac{c}{\sigma|z|}},
$$

where $\sigma$ is the pipe wall conductivity. Taking into account Eqs. (5) and (17), one can represent the normalized wake function as $q=q_{0} w(\theta)$ with

$$
q_{0}=-\frac{4 r_{0} R^{2} N_{b}}{3 \pi \gamma \beta b^{3} Q_{0}} \sqrt{\frac{c}{\sigma z_{b}}}, \quad w(\theta)=\frac{3}{4 \sqrt{2 \theta}} .
$$

Then series (19) is applicable with the matrix $R_{N, n}$ whose part is represented in Table II. Note that $R_{0,0}=1$ due to the special choice of the coefficients in Eq. (32). The threshold of this instability is plotted with different approximations in Fig. 8, which is very similar to Fig. 5 (constant wake) both in the form and in the magnitude.
Generally speaking, the resistive wake can cause multiturn collective effects as well. However, their influence on the TMCI threshold is negligible for a single bunch at the condition $z_{b} \ll 2 \pi R$ [5].

\section{B. Short square wake}

A square wake can be created by a strip-line beam position monitor or by a traveling-wave kicker [2]. The long (constant) square wake has been considered above in detail. However, the wake can be shorter than the bunch, in practice. In accordance with Eq. (17), its normalized strength should be represented in the form

$$
q(\theta)=q_{0} w(\theta)=\frac{4 q_{0}}{\theta_{w}\left(4-\theta_{w}\right)} \quad \text { at } 0<\theta<\theta_{w}
$$

where $\theta_{w}<2$ is the wake length (recall that the bunch length is 2 in these units). Several examples are represented in Fig. 9 at $n_{\max }=9$. Note that the horizontal lines have no physical sense and are added to mark the end of the curve applicability (the calculations were not carried out after that). Because of the normalization, the threshold dependence on the SC tune shift is not very significant, especially

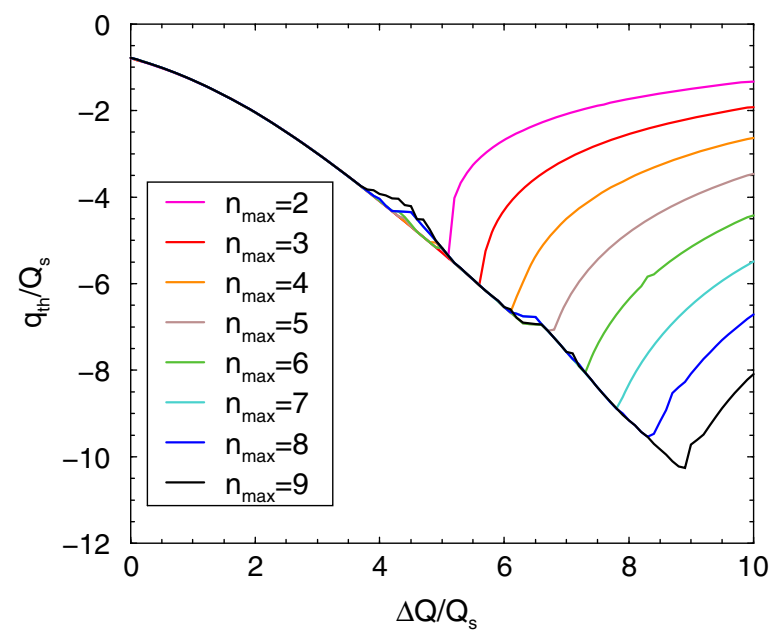

FIG. 8. Stability region of the boxcar bunch with a resistive wall wake. The normalized magnitude of the wake is given by Eq. (32), and index $n_{\max }$ means maximal power of the Legendre polynomial in the expansion. The left-hand line shows the TMCI threshold, and the right-hand (rising) curves are unphysical. 


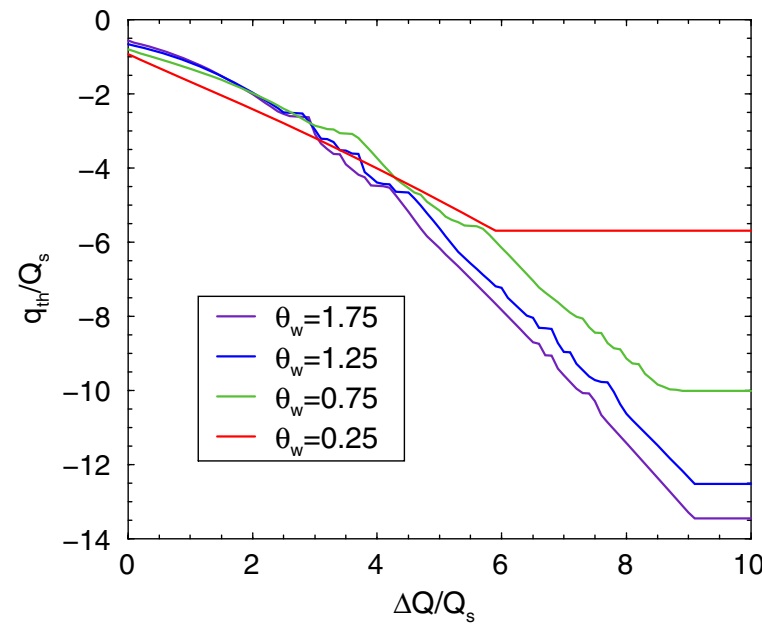

FIG. 9. TMCI threshold of a short square wake against a SC tune shift. The length of the wake is $\theta_{w}$, and the bunch length is 2 . The approximation with $n_{\max }=9$ (55 modes) is used; the horizontal lines mark the end of the applicability area.

at $\Delta Q / Q_{s}<5$. However, the area of applicability of the result decreases at the wake shortening.

\section{Oscillating wake}

There are several models considering the wakefield source as a resonator of frequency $f=c / \lambda$ [2]. It creates an oscillating wake $\propto \cos (2 \pi z / \lambda)$ having the phase advance $\phi=2 \pi z_{b} / \lambda$ within the bunch. We consider the case $\phi<2 \pi$ and represent the wake in the form satisfying the normalization condition (17):

$$
q(\theta)=q_{0} w(\theta), \quad w(\theta)=\frac{\phi^{2} \cos (\phi \theta / 2)}{2(1-\cos \phi)} .
$$

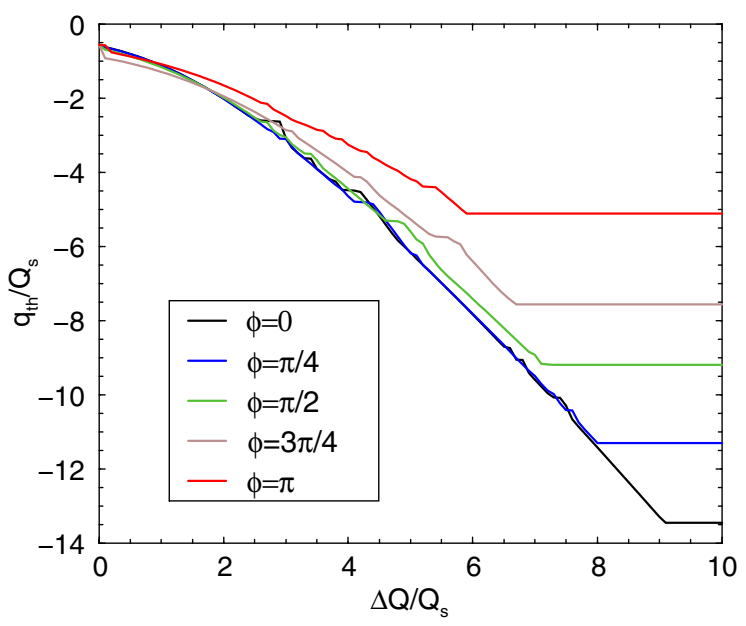

FIG. 10. TMCI threshold of an oscillating wake with phase advance $\phi$ within the bunch of length $\Delta \theta=2$. The approximation with $n_{\max }=$ (36 modes) is used; the horizontal lines mark the end of the applicability area.
Several examples are represented in Fig. 10 at $n_{\max }=7$ to demonstrate that SC produces a stabilizing effect in these cases as well.

\section{CONCLUSION}

Being stable in themselves, the eigenmodes of the boxcar bunch with space charge form a convenient and effective basis for the investigation of the bunch instability with the space charge and wakefields. A series of equations is derived in this paper using this expansion technique. For a constant wake, the dispersion equation is represented in the form of an infinite continued fraction as well as in the form of a recursive relation with an arbitrary number of basis functions involved.

It is shown that the TMCI threshold of the negative constant wake grows in absolute value when the SC tune shift increases. An enlargement of the number of the used basis vectors expands the area of applicability of this statement but does not change the results obtained before. This statement is confirmed in this paper by the straightforward calculation of the threshold at $\Delta Q / Q_{s} \leq 12$ using the basis set including up to 91 eigenfunctions. A very good convergence of different approximations is obtained in this region. However, the convergence of the solutions is not achieved at a higher $\mathrm{SC}$, because a very large number of eigenfunctions is required for the separation of different radial modes. Nevertheless, an additional analysis of the bunch spectrum allows one to extend the statement to any tune shift.

Similar results are obtained with realistic wake functions including the resistive wall, the short square, and the oscillating forms. A monotonic increase of the TMCI threshold with a SC tune shift has been demonstrated earlier by Blaskiewicz, who was using the model of a hollow bunch in a square potential well with an exponential wake [3].

The threshold of the positive wake goes down when the SC tune shift increases, and the effect can be satisfactorily described by the three-mode approximation.

\section{ACKNOWLEDGMENTS}

Fermi National Accelerator Laboratory is operated by Fermi Research Alliance, LLC under Contract No. DEAC02-07CH11395 with the United States Department of Energy.

\section{APPENDIX: CALCULATION OF THE NORMALIZING COEFFICIENTS AT EQ. (13)}

Using the notation

$$
\begin{aligned}
\hat{\nu}_{n, m} & =\frac{\nu_{n, m}+\Delta Q}{Q_{s}}, \quad \hat{\Delta} Q=\frac{\Delta Q}{Q_{s}}, \\
P_{n}(\theta) & =\sum_{l=0}^{n} p_{n l} \theta^{l},
\end{aligned}
$$

one can rewrite Eq. (13) in the form 
$\hat{\nu}_{n, m} Y_{n, m}+i \frac{\partial Y_{n, m}}{\partial \phi}=S_{n, m} \Delta \hat{Q} \sum_{l=0}^{n} p_{n, l}(A \cos \phi)^{l}$.

Its solution is

$$
Y_{n, m}=S_{n, m} \Delta \hat{Q} \sum_{k=-n}^{n} \frac{\exp i k \phi}{\hat{\nu}_{n, m}-k} \sum_{j=0}^{n} U_{n, k, j} A^{k+2 j},
$$

where

$$
U_{n, k, j}=\frac{p_{n, k+2 j}}{2^{k+2 j}}\left(\begin{array}{c}
k+2 j \\
j
\end{array}\right) \times\left\{\begin{array}{l}
1 \text { at } k+j \geq 0, \\
0 \text { at } k+j<0 .
\end{array}\right.
$$

This function should satisfy the normalization condition represented by Eq. (7) with $j \equiv\{n, m\}$ and distribution function (11a). The substitution results in the relation

$$
\begin{aligned}
\frac{1}{S_{n, m}^{2} \Delta \hat{Q}^{2}}= & \sum_{k=-n}^{n} \frac{1}{\left(\hat{\nu}_{n, m}-k\right)^{2}} \\
& \times \sum_{j_{1}=0}^{n} \sum_{j_{2}=0}^{n} U_{n, k, j_{1}} U_{n, k, j_{2}} \overline{A^{2\left(k+j_{1}+j_{2}\right)}},
\end{aligned}
$$

where $\overline{A^{2 j}}$ is the amplitude power averaged over the distribution:

$$
\overline{A^{2 j}}=\int_{0}^{1} \frac{A^{2 j+1} d A}{\sqrt{1-A^{2}}}=\sum_{l=0}^{j}\left(\begin{array}{l}
j \\
l
\end{array}\right) \frac{(-1)^{l}}{2 l+1} .
$$

In principle, involved eigentunes $\nu_{n, m}$ could be obtained by the substitution of Eq. (A3) into Eq. (4) with the functions $Y$ and $\bar{Y}$ being taken from this Appendix. Because a similar calculation has been actually accomplished in Ref. [9], we represent here only the resulting equation for the eigentunes:

(i) Lower powers.-

$$
\hat{\nu}_{0,0}=\Delta \hat{Q}, \quad \hat{\nu}_{1, \pm 1}^{2}-1=\Delta \hat{Q}_{\hat{\nu}_{1, \pm 1}} .
$$

(ii) Higher even powers.-

$$
\begin{aligned}
& \hat{\nu}_{n, m}\left[\hat{\nu}_{n, m}^{2}-4\right] \ldots\left[\hat{\nu}_{n, m}^{2}-n^{2}\right] \\
& \quad=\Delta \hat{Q}\left[\hat{\nu}_{n, m}^{2}-1\right] \ldots\left[\hat{\nu}_{n, m}^{2}-(n-1)^{2}\right] .
\end{aligned}
$$

(iii) Higher odd powers.-

$$
\begin{aligned}
& {\left[\hat{\nu}_{n, m}^{2}-1\right] \ldots\left[\hat{\nu}_{n, m}^{2}-n^{2}\right]} \\
& \quad=\Delta \hat{Q} \hat{\nu}_{n, m}\left[\hat{\nu}_{n, m}^{2}-4\right] \ldots\left[\hat{\nu}_{n, m}^{2}-(n-1)^{2}\right] .
\end{aligned}
$$

Some of the values $\nu_{n, m} / Q_{s}=\hat{\nu}_{n, m}-\Delta \hat{Q}$ are plotted in Fig. 1 . Factors $S_{n, m}^{2}$ can be found from Eq. (A5) with the known eigentunes substituted. Some results are represented below:

$$
S_{0,0}^{2}=1, \quad S_{1, \pm 1}^{2}=\frac{3 \hat{\nu}_{1, \pm 1}^{2}}{\hat{\nu}_{1, \pm 1}^{2}+1}
$$

$$
S_{2, m}^{2}=\frac{5\left(\hat{\nu}_{2, m}-1\right)^{2}}{\hat{\nu}_{2, m}^{4}+\hat{\nu}_{2, m}^{2}+4}, \quad m=2,0,-2,
$$

$$
S_{3, m}^{2}=\frac{7 \hat{\nu}_{3, m}^{2}\left(\hat{\nu}_{3, m}^{2}-4\right)^{2}}{\hat{\nu}_{3, m}^{6}-2 \hat{\nu}_{3, m}^{4}+13 \hat{\nu}_{3, m}^{2}+36},
$$

etc.

Solutions of the second equation in Eq. (A7) are

$$
\begin{aligned}
& \hat{\nu}_{1, \pm 1}=\frac{\Delta Q}{2} \pm \sqrt{\frac{\Delta Q^{2}}{4}+1}, \\
& \nu_{1, \pm 1}=-Q_{s} \hat{\nu}_{1, \mp 1} .
\end{aligned}
$$

Applying it to the second equation in Eq. (A10), we obtain

$S_{1,1}^{2}+S_{1,-1}^{2}=3, \quad \hat{\nu}_{1,1} S_{1,-1}^{2}+\hat{\nu}_{1,-1} S_{1,1}^{2}=0$.

The relation for the coefficient $W_{1}(\nu)$ follows from this:

$$
\begin{aligned}
W_{1}(\nu) & =\frac{\left|S_{1,1}\right|^{2}}{\nu-\nu_{1,1}}+\frac{\left|S_{1,-1}\right|^{2}}{\nu-\nu_{1,-1}} \\
& =\frac{3(\nu+\Delta Q)}{\nu(\nu+\Delta Q)-Q_{s}^{2}},
\end{aligned}
$$

which expression is used in the main text as Eq. (24).

[1] R. Kohaupt, Transverse instabilities in PETRA, in Proceedings of the XI International Particle Accelerator Conference, Geneva, Switzerland, 1980 (CERN, Geneva, Switzerland, 1980), p. 562; DESY Report No. M-80/19, 1980.

[2] K. Y. Ng, Report No. Fermilab-FN-07-13, 2002.

[3] M. Blaskiewicz, Fast head-tail instability with space charge, Phys. Rev. ST Accel. Beams 1, 044201 (1998).

[4] M. Blaskiewicz, Comparing new models of transverse instability with simulations, in Proceedings of the 3 rd International Particle Accelerator Conference, New Orleans, LA, 2012 (IEEE, Piscataway, NJ, 2012), p. 3165.

[5] V. Balbekov, Single bunch transverse instability in a circular accelerator with chromaticity and space charge, J. Instrum. 10, P10032 (2015).

[6] A. Burov, Head-tail modes for strong space charge, Phys. Rev. ST Accel. Beams 12, 044202 (2009).

[7] V. Balbekov, Transverse instability of a bunched beam with space charge and wakefield, Phys. Rev. ST Accel. Beams 14, 094401 (2011).

[8] V. Balbekov, Report No. FERMILAB-PUB-16-079-APC, 2016.

[9] F. Sacherer, CERN Report No. SI-BR-72-5, 1972.

[10] H. G. Hereward, CERN Report No. MPS-Int-DL-64-8, 1964.

[11] V. I. Balbekov and K. F. Gertsev, Beam coherent instability in IHEP accelerator, At. Energ. 41, 408 (1975). 